ESTUDIOS

\title{
Justicia, familia y cuidado: Apuntes normativos en políticas públicas
}

\author{
Justice, family and care: Notes on public policies
}

\author{
Vinicius Ferreira Baptista \\ Universidad Federal Rural de Río de Janeiro, Brasil
}

\begin{abstract}
RESUMEN Este trabajo analiza las cuestiones de género relacionadas con la familia y el cuidado desde perspectivas teóricas y contribuye con datos y propuestas de políticas públicas organizadas en los últimos años en Brasil. Discute los aportes críticos de Susan Moller y Christine Delphy y los contraargumentos de John Rawls, Ronald Dworkin y Nancy Fraser. Incorpora al debate las propuestas legislativas de la Cámara de Representantes. A modo de conclusión, se postula que las propuestas legislativas deben abarcar el cuidado y la familia en su dimensión de género, raza y clase, a través de mecanismos claros que refuercen una perspectiva social de justicia y desarrollo que apunten hacia sujetos de justicia y derechos humanos; al mismo tiempo que incidan en el potencial de los individuos.
\end{abstract}

PALABRAS CLAVE Género, familia, cuidado, políticas públicas, justicia.

ABSTRACT This paper analyses gender issues in domestic work and care from theoretical perspectives, contributing to the data and proposals of public policies organized in the last years in Brazil. We discussed the critical contributions of Susan Moller and Christine Delphy and the counterarguments of John Rawls, Ronald Dworkin and Nancy Fraser. We bring to the debate legislative proposals from the Chamber of Representatives. In conclusion, we consider that the legislative proposals should include care and domestic work in its gender, race and class dimensions, through clear mechanisms that enhance a social perspective of justice and development that add up to subjects of justice and human rights; the same time that affects the potential of individuals.

KEYWORDS Gender, family, care, public policy, justice. 


\section{Introducción}

En la sociedad existen relaciones de producción y reproducción; según Delphy (2016: 29) los análisis tradicionales de este proceso centran sus esfuerzos en el primer sistema, la producción, olvidando su esencia y situándolo como reproductivo. La autora destaca la necesidad de analizar la participación de las mujeres en la producción de la familia tal como sucede, por ejemplo, con las actividades productivas que tienen una función económica. Vista así, la familia pasa a ser un lugar de expropiación de las mujeres, puesto que la asignación de responsabilidades no remuneradas en las actividades domésticas y el cuidado de los niños se asemejan a la esclavitud.

Por su parte, Moller entiende como una de las fuentes centrales de injusticia para las mujeres el trato, ante la ley, más o menos igualitario para aquellos que pasan por procesos desiguales en diferentes esferas de la vida. Uno de estos procesos sucede en la estructura familiar y su aparente toma de decisiones «consensuadas» $\mathrm{O}$ «racionales» sobre asuntos en los que el elemento financiero de mayor renta del marido, prácticamente, guiaría la decisión. El matrimonio, según la misma autora, estructurado de acuerdo a patrones de género, hace a las mujeres vulnerables porque las sociedades ven las características «innatas» del sexo como legitimadoras de diferentes derechos que implican restricciones formales e informales, institucionalizando la diferencia sexual (Moller, 1989: 5). Y en este punto habría una crisis significativa de justicia que guiaría las cuestiones de género.

Así, nuestro objetivo es analizar las cuestiones de género, familia, trabajo doméstico y cuidado, desde perspectivas teóricas y, además, aportar algunos datos y propuestas de políticas públicas organizadas en los últimos años en Brasil. En primer lugar, presentamos las consideraciones críticas de Susan Moller y Christine Delphy con relación a las perspectivas de género, familia y trabajo; dentro de una coyuntura de justicia (en donde Moller se aleja de las perspectivas liberales clásicas, mientras Delphy, se aleja de la óptica reificadora de género y clase). A continuación, señalamos los contraargumentos de John Rawls, Ronald Dworkin y Nancy Fraser, en donde destacamos sus diferentes análisis de la justicia y los modelos normativos añadidos al cambio (o no) de la realidad social. Concluimos con proyectos de ley en el ámbito de la Cámara de Diputados, situando la densa coyuntura socialmente injusta para las mujeres, así como la clara neutralidad de género en las propuestas de política pública, lo que perjudica la construcción de una sociedad más justa e igualitaria.

\section{Las críticas de Susan Moller y Christine Delphy}

Todas las sociedades contemporáneas dependen del trabajo no remunerado de la mujer en el cuidado y los servicios domésticos que se desarrollan dentro de una relación privada. Allí las mujeres quedan excluidas de las relaciones de intercambio y 
no son valoradas — financierizadas, en términos de Delphy (2016: 47-49) —, puesto que el retorno tiene lugar en forma de regalos o agrados del marido, cuya responsabilidad es mantener su poder de trabajo. No es la naturaleza de las actividades, sino las relaciones de producción las responsables de la exclusión de las actividades del ámbito del valor.

La familia es una unidad de producción donde la explotación aún persiste, ya que se basa en relaciones consanguíneas o por matrimonio. La «ayuda» de la familia se institucionaliza en las estadísticas, en vista de que sus retornos van especialmente dirigidos al jefe de esta (Delphy, 2016: 64-65). Los servicios domésticos desarrollados por las esposas no son diferentes de los otros bienes y servicios producidos y consumidos por la familia; así, no hay distinción entre el valor del bien y el intercambio. En ese sentido, nos preguntamos: si el servicio doméstico no hubiera sido producido y continuase la demanda, ¿se percibiría su valor de uso y de intercambio?

La omisión de la familia, así como la falsa neutralidad de género en el lenguaje, instalarían la negligencia política en la cuestión de género. Las mujeres no están contempladas en las teorías, la igualdad de oportunidades se erige sobre desigualdades construidas y la familia, además de no ser incluida, no tiene una forma justa, cuyas relaciones apunten al desarrollo moral (Moller, 1989: 13-14). Lo que vemos es que la falsa neutralidad de género invisibiliza las condiciones de la injusticia, debido a que no señalar claramente cómo se componen las relaciones de desigualdad impide afirmar la dimensión de la injusticia. E ignorar la dimensión política de las relaciones de justicia es trasladar la justicia como un problema político. Siguiendo a Hume (1967), donde no hay desigualdad, ni la disputa por elementos que caracteriza a la escasez, no existe demanda por justicia: negar las condiciones y desalentar a los sujetos de la justicia es negar la justicia misma.

Desde la perspectiva de la justicia y la familia idealizada, Moller (1989: 26-29) revisa la noción de Rousseau y Hume que caracteriza a esta última como un espacio impregnado de afecto y amor, donde no existe prerrogativa acerca de lo que es justo, ya que el elemento político de la justicia no puede ser auditable ni dividido en partes cuantitativas para ser asignadas individualmente. Aquí la virtud del amor prevalece a la racionalidad de la justicia. En la familia no hay disputa por los bienes ni recursos; por el contrario, es solo benevolencia, lo que la vuelve inmune a los sentimientos dañinos e inmorales que desvirtúan a las personas a la corrupción de la justicia. Hume aclara esta cuestión al proponer que la familia se construye como un lugar donde la justicia no sería discutida, porque no es un territorio de invocación.

En esta función, el sacrificio por la familia, o su cuidado intergeneracional y la vida íntima - tres íconos culturales de reciprocidad-, son movilizados por la lógica del mercado y gradualmente transformados (Araujo, 2019: 2-4). Así se fragua la eliminación de identidades personales y colectivas a través de la obligación moral de las responsabilidades familiares en la construcción de este trabajo ejercido, en su 
mayoría por las mujeres, en una idealización de estas funciones femeninas de cuidado como no remunerado. La reciprocidad, el impulso de la preocupación misma, es convertido en una obligación moral que, indirectamente, fundamenta el trabajo de cuidado en las familias y en el mercado.

Para Hume (1967: 243-244), la justicia es un proceso civilizador. Metodológicamente, su objeto recae en los bienes (partiendo por los privados y avanzando hacia los públicos): su origen conceptual, su escasez y la necesidad de compartir su motivo dentro de una sociedad que implica experiencia mutua. En una situación de abundancia no hay salvaguardia de la justicia y esta no se invoca. La justicia se perfecciona a través de una medida de salvaguardia que reestructura la situación en la que, debido a la libertad y al libre albedrío, los hombres optan por no seguir ciertas reglas morales establecidas en la vida social y que permanecen históricamente; y las bases morales son volubles, pueden desencadenar pasiones desenfrenadas en varios momentos.

Cuando ciertas características humanas - la benevolencia, por ejemplo- no producen la acción y el sentimiento correcto en ciertos individuos, es probable que estos no respeten la construcción justa de reglas morales (dirigidas al beneficio del individuo y a la utilidad social). Al romper con la solidaridad, no necesitan permanecer en línea con el respeto y avanzan a la inestabilidad; impulsan, así, la reciprocidad en las acciones de los demás, porque una vez que se interrumpe el proceso que, generalizado, se extiende y produce una necesidad extrema como base, sitúa la ruptura total de cualquier norma moral y del derecho. Por lo tanto, la sociedad necesita ser preservada de estos ruidos e inestabilidades.

En consecuencia, la familia estaría más allá de la justicia para este autor, ya que los roles que a ella se dan y su objetivo final no implica la percepción de utilidad. La familia sería necesariamente el lugar de la correcta acción y el sentimiento humanitario y, por ello, de la construcción de normas justas. El mantenimiento de la estructura familiar y la crianza de los hijos son el fin objetivo de este modelo que trae bases morales independientes de la responsabilidad que producen inestabilidad social; y la familia no participa en esta inestabilidad. Considerando que es un modelo cerrado en sí mismo, termina pasando lo contrario en la realidad compleja, donde la inexistencia de la familia es el factor que implica esta inestabilidad, en que vemos el fortalecimiento del modelo familiar como estructuración de la sociedad en el tiempo y en el espacio. Los bienes y recursos familiares son para la propia familia, así que, si todo el mundo los disfruta, ¿por qué considerar útil o injusta la distribución? No hay terreno para evocar justicia y lo que sucede en estos términos es enterrado en la propia estructura. La moral también pasa por esta misma situación descrita.

La idea de la familia como un espacio inalcanzable por disputas, jerarquías y desigualdades idealiza las instituciones e interfiere en la perspectiva de la justicia al no considerar beneficios, cargas ni elementos distributivos verificables. Para Moller (1989: 92-93), el espíritu de generosidad impregna las relaciones en la familia 
de forma que la primacía de la justicia es vista como esencial para la fundación de la misma. La perspectiva de la familia injusta como natural y necesaria se basa en la sujeción de las mujeres y la división sexual del trabajo. La mujer que no sigue este camino de la familia representa el desorden y la ruina de la sociedad porque amenazaría con destruir una estructura primordial de ella, lo que ilustra el fuerte carácter biologizador, patológico y moralizador en la naturalización de la injusticia a través de la familia. Moller sitúa a la corriente comunitaria, argumentativamente, como reaccionaria y desigual, señalando que destaca un retorno a la tradición y los entendimientos mutuos como sus bases, en las que no incluyen a las mujeres como sujetos plenos de justicia. En particular, por el uso de un lenguaje neutral en cuanto al género en el debate de las tradiciones, que no tiene en cuenta la desigualdad. Surge la interrogante acerca de cuál es la racionalidad evocada aquí.

En Rawls (1971: 13-25) vemos un claro proceso de términos masculinos como referencia, especialmente en vista de los elementos kantianos y freudianos en la construcción teórica (Moller, 1989: 92). La ambigüedad en el uso de los términos no se enfrenta en la posición original y uso del velo de la ignorancia no observa la estructura generificada de la sociedad que produce desigualdad de género. El velo de ignorancia de Rawls (1971: 165-173) implica un modelo hipotético en el que personas consideradas iguales realizan la toma de decisiones de manera racional basada en una dinámica en la que la información de los tomadores de decisiones no está disponible. El objetivo es delimitar la toma de decisiones justo en el sentido de que quien decide comprende que su decisión le puede afectar al ignorar las condiciones sociales que involucran a ambos (ya que solo se ofrece una mínima información circunstancial).

Aun así, Rawls (1993: 257-288) excluye la estructura familiar en la estructura básica de la sociedad, debido a que es un elemento que genera efectos y guía las relaciones. El esquema rawlsiano no considera sujetos moral, legal o socialmente degradados; el autor simplemente los excluye en su modelo, al considerar como representantes a personas libres, racionales e iguales. Es decir, incluir al «degradado» destruye por completo el esquema rawlsiano, porque fuerza la ruptura con los individuos «normales» que no tendrían ningún contexto de degradación para la toma de decisiones; alguien considerado «degradado» podría tomar una decisión a la luz de su condición, pues es información que va más allá del velo de la ignorancia por ser parte del individuo.

En particular, hay una «condición humana» en el modelo de Rawls que no es biológica, sino que relacionada a ciertas capacidades. El desafío es planteado por Nussbaum (2006: 18-27), quien rechaza tal «condición humana» definida únicamente por ciertas capacidades y señala el problema potencial: excluir del sentido de lo «humano» a quienes no cumplan con ellas. La autora desafía el modelo contractualista de Rawls y los principios de Dworkin (1985: 105-127), entendiendo que son modelos agotables en sí mismos, por localizar agentes racionales, libres y autónomos 
como los principios que organizan la sociedad. El punto es cómo considerar a las personas que tienen brechas de capacidad mental, a los animales y los apátridas; los tres considerados distantes en la lógica «humana», cada uno con sus justificaciones de negligencia de los derechos.

La familia aparece en tres contextos para Rawls (1993: 456-472), como: 1) conector intergeneracional; 2) obstáculo a la igualdad de oportunidades; y 3) primera escuela de desarrollo moral. Además, según Rawls, la familia, como institución, no se configura como un espacio político de disputas, por lo que todos aquellos más allá del velo de la ignorancia serán participantes en el mercado laboral remunerado. Rawls asume a la familia como una asociación privada cualquiera. Aquí, a diferencia de Hume, los individuos que la componen tienen metas y planes de vida propios y, sin embargo, por tener un sentido de justicia y una visión de bien común, pueden ajustarse a la estructura social que los vincula. Volveremos a Rawls más tarde.

La familia es una asociación que desarrolla la comprensión moral; el sentido de equidad provendría del aprendizaje producto del contacto con diferentes perspectivas, ya que es esencial para la otredad propuesta en el velo de la ignorancia. En otras palabras, la familia involucra un conjunto de información que afecta las estructuras y planes de vida de las personas, por lo tanto, en la lógica rawlsiana, deben estar contenidas en la base del velo de la ignorancia. Moller (1989: 184) señala que, si en realidad vemos la generificación de la estructura familiar desarrollar moralmente a los niños, ¿cómo situar la justicia moral y la equidad en el desarrollo? La misma autora defiende la idea de aplicar la posición original y el velo, y que es necesario considerar a las mujeres en la perspectiva generificada; así como a las familias dentro de los representantes y «jefes de familia representativos». Además, debe considerarse la división sexual del trabajo y la remuneración del trabajo no pago y quién ocupa estas posiciones. Los factores sociales deberían ser considerados por instituciones sin género para que el ideal de rawlsiano funcione.

El consumo y su distribución, así como las dimensiones de representación y producción apuntan a mecanismos de posición social coercitivos y vergonzosos, acceso a bienes y situación en el orden socioeconómico (Delphy, 2016: 52-55). Este proceso se estructura en las funciones de esposa y madre como «gerentes» de la casa, siendo la mujer restringida en las decisiones y opciones relacionadas. Por lo tanto, las experiencias variarán según la edad, la cantidad de hijos, la ocupación de la mujer y sus ingresos, en caso de tenerlos; así como el trabajo del marido y la posición de la familia. La situación de clase está relacionada con las dimensiones de acceso, distribución y consumo en este orden familiar.

Existe un continuum entre la producción y el consumo en las familias, en el que el proceso de la primera no se ve como un servicio especializado, por lo que no es comercializable; así no se interrumpe la parálisis de la no valorización del servicio (Delphy, 2016: 65-67). Si no se produce, este se compra en su forma final. Si es venta- 
joso impregnar la producción (por obra no remunerada), los bienes intermedios se compran para proceder a la producción y el consumo final; como ingredientes para la preparación de una comida o los tejidos y acciones para la fabricación de tejidos. Cualquiera que sea la naturaleza de la obra producida dentro de la familia, ella no será remunerada.

Aunque la división sexual del trabajo no está en el análisis de El capital, es posible utilizarlo para la opresión de las mujeres (Delphy, 2016: 159). Hay que considerar la distinción entre la persona que recibe el servicio y la que incorpora la obra. Este punto es fundamental, porque dentro de una estructura familiar, además del marido y la esposa, pueden existir otros parientes, como los hijos; aquí, ¿los niños serían directos apropiadores del trabajo de las mujeres, usurpándoles tiempo y esfuerzo, sin pagar por su trabajo? La incorporación del trabajo es en relación con aquel que se beneficia directamente del servicio prestado y, que, además, por no ser remunerado vuelve bajo agrados, e incorpora a su recuperación de la fuerza de trabajo a través de la fuerza de trabajo de la mujer; esto no sucede con los niños y los ancianos, por ejemplo, que no se incorporan a su actividad productiva.

Aquí el movimiento feminista obliga al reconocimiento del trabajo doméstico como trabajo, además, explotado (Delphy, 2016: 174). La explotación de las mujeres no puede ser trasladada del campo económico y político hacia el campo cultural, en el que la opresión es un factor meramente ideológico; así, se sitúa el potencial materialista que deja claro el mayor beneficiario de este proceso: el hombre. Este traslado del ámbito económico y político hacia lo cultural refuerza las bases «históricas», en las que la sujeción de la mujer concatena el orden social, articulando los intereses de la familia y de la sociedad como si no tuvieran ninguna relación más allá del alcance de la «casa». Esta posibilidad materialista incorpora la dimensión dialéctica e histórica de un proceso de acumulación y apropiación de la fuerza de trabajo que no se contabiliza en el sistema productivo y que construye no solo la reducción socioeconómica, sino también política e institucional que guía las diferentes esferas de la vida.

Una teoría de la justicia no puede ignorar la dicotomía entre lo público y lo doméstico. Algunas de ellas separan esta dualidad en esferas. No necesariamente cada esfera es independiente o responde sistemáticamente al efecto directo en el ámbito político y social, en el sentido de que son capaces de alterar las estructuras sociales de organización, oportunidades, acceso y relaciones sociales. En este punto, la familia, aisladamente, no puede considerarse una esfera, sino que refleja, sobre la cuestión de la desigualdad, de la exclusión de otras esferas (Moller, 1989: 115). Además, el trabajo no remunerado como un componente de las estructuras de desigualdad en la familia, no es considerado como una esfera que produce y refleja desigualdades. Por lo tanto, una de las críticas de Moller a la noción de «lo personal es político», entiende que una esfera privada personal y el concepto de privacidad son importantes para las mujeres, 
desde que los miembros sean tratados de manera justa e igualitaria hasta que aquellos considerados desiguales estén protegidos del abuso.

Cuando las mujeres casadas trabajan y ganan salario, además proporcionan condiciones, mientras que la mujer casada sin trabajo realiza el servicio doméstico, que justifica su subsistencia y la de la casa. Delphy (2016: 70-71) considera que, en el primer caso, el trabajo remunerado de las mujeres casadas y su esfuerzo productivo no habrá servido a la justificación de nada, ya que sigue siendo responsable de los servicios domésticos. Para la autora, el modelo familiar de producción da lugar a la explotación familiar o patriarcal (Delphy, 2016: 78-83) y el trabajo de la mujer es apropiado, total o parcialmente, por la familia y esto no entra en la lógica de los costos.

Moller coloca el matrimonio centrado en la generificación de roles y posiciones como el lugar de poder y la vulnerabilidad a la dependencia, la explotación y el abuso. En este campo, la vulnerabilidad, socialmente inscrita (y física, también en mi opinión), configura la jerarquía y guía las bases para la construcción de la desigualdad (Moller, 1989: 136-138). Este caso se aproxima al argumento de Fraser (2013: 111-115) respecto a que la vulnerabilidad no debe significar degradación en la actividad social. La vulnerabilidad se produce históricamente y en círculos, es decir, se reorienta con el refinamiento de las prácticas y la construcción argumentativa.

En este punto vemos que la dimensión de los ingresos tiene una fuerte asociación con el poder y la toma de decisiones dentro del matrimonio. El matrimonio, el trabajo doméstico y el trabajo remunerado reúnen dimensiones que situarán la vulnerabilidad de las mujeres (Moller, 1989: 159). La distribución y el ejercicio del poder dentro de la familia y sus ramificaciones más allá de ella se manifiestan como un círculo vicioso. La familia es el motor del género, reproduciéndolo entre generaciones. Crea expectativas y guía la vida de las mujeres, haciéndolas vulnerables (Moller, 1989: 173). Para una teoría de la justicia, la familia necesita ser una institución justa. Aquí, el trabajo reproductivo debe ser reconocido como trabajo y tener una remuneración justa.

Para Delphy, el matrimonio es un estado de autopercepción de las mujeres - el estancamiento interfiere con sus planes de vida a corto y largo plazo, especialmente aquellos relacionados consigo mismas, como el estudio y el trabajo- donde vemos brechas socioeconómicas en términos de ocupaciones y oportunidades de trabajo y logros salariales (Delphy, 2016: 98). En caso de divorcio, vemos a las mujeres entrando en un contexto de baja cualificación y experiencia, así como asociadas con empleos mal pagados. Sin embargo, Delphy (2016: 93-105) señala el divorcio como continuación del matrimonio, ya que la responsabilidad de los hijos continúa con la mujer, incluidos sus costos, que no son totalmente absorbidos, por ejemplo, con pensiones pagadas por sus exmaridos.

La responsabilidad dentro de la familia debe ser paterna y no monológica por parte de la madre, con instalaciones públicas y políticas de cuidado, y esto debe alcanzar la esfera del trabajo con garantías de estabilidad (Moller, 1989: 178-175). El trabajo do- 
méstico es un trabajo productivo por incidir en los costos sociales y económicos de la disposición de la fuerza de trabajo, su reproducción y recuperación de su fuerza para la producción, lo que interfiere con el sistema productivo en su conjunto (Dephy, 2016: 87-89). El trabajo doméstico no se paga ni se intercambia en formas comunes en el mercado, en lógicas de oferta y demanda, ni en ajustes de costos y formas de producción. Las relaciones sociales y matrimoniales configuran un contrato que desfavorece a la esposa trabajadora. Si ella no es remunerada, está siendo apropiada por otros, estableciendo una relación de producción. En este punto Delphy (2016: 90-92) deja de utilizar el término tareas domésticas para incorporar el término trabajo familiar para asociar a la familia en su forma política, productiva y relacional.

\section{La ¿respuesta? de John Rawls}

Moller señala que los términos de la justicia creados después de la década de 1960 continúan viendo a las mujeres superficialmente, especialmente cuando tratan a la familia como no política (Moller, 1989: 9), y que los autores asumen esta negligencia porque entienden a las mujeres como sujetos morales, donde la idea de independencia y trabajo remunerado dictan bases de reconocimiento sustantivo para la teorización. Sin embargo, las ideas desarrolladas por estos autores impregnarían una neutralidad de género, es decir, que este reconocimiento que implica independencia y trabajo no tendría interferencia o sobreposición masculina en su construcción.

Con el fin de situar las mejores formas de realizar los valores políticos fundamentales de la libertad y la igualdad, De Vita (1993: 35-37) subraya que los principios de la justicia deben organizarse de acuerdo con la resolución de conflictos de las circunstancias objetivas de la justicia (Rawls, 1971: 65-72). Es una concepción pública de la justicia, moralmente justificada para cada uno de los ciudadanos, especialmente aquellos que están peor situados en el momento, para fines de reivindicación de acciones públicas.

El concepto de justicia practicable presupone sociedades democráticas basadas en: 1) pluralismo político y moral; y 2) la autopercepción de los individuos como personas morales, libres e iguales. Esta concepción es abstracta ya que se concentra en elementos que establecen restricciones a la naturaleza de la justicia en la sociedad y sirve, también, como criterio para la evaluación del grado de justicia, para De Vita (1993: 39).

De Vita busca analizar el principio de la diferencia, considerando que Rawls asume como presunción la idea de que un individuo no puede ser perjudicado por accidentes sociales o genéticos en sus elecciones dentro de una sociedad bien ordenada (De Vita, 1993: 47-48). Por lo tanto, el problema de la justicia es definir que las desigualdades pueden justificarse, teniendo en cuenta la distribución equitativa y aceptada de las desigualdades de acuerdo con el criterio de la justicia que tiene 
por objetivo la mejora sustancial de quienes se encuentran en peores situaciones en la sociedad. Lo que es justo o injusto, sería cómo las instituciones de la sociedad se ocuparían de los hechos en la sociedad (hechos como la posición social, el género, la raza, la clase, las habilidades, la genética, el azar, las debilitaciones, entre otros). Por lo tanto, el objetivo sería la neutralización de los efectos de la distribución natural de estos procesos.

Rawls no consideró a la familia en obras como Theory of justice o Political liberalism. Sin embargo, el autor no hizo caso omiso de las críticas de Moller y las trabajó, parcialmente, en su libro Justice as fairness, donde señala a la familia como una institución básica de la estructura de la sociedad. Si bien Rawls murió antes de terminar este libro, el análisis, aunque incompleto, logra evidenciar que tenía como objetivo considerar al menos tres elementos que involucran a la familia: 1) las diferentes formas familiares que deben ser aceptadas legal y socialmente; 2) el cuidado de los niños y su desarrollo moral para la institucionalización intergeneracional de los principios de la justicia; 3) la desproporción histórica de la división sexual del trabajo entre hombres y mujeres en el ámbito familiar.

La familia forma parte de la estructura básica de la sociedad y su función es establecer la producción y reproducción de la misma, dándole una base cultural intergeneracional, especialmente a partir del desarrollo moral y la educación cultural integral de los niños, que forma su sentido de justicia y virtudes políticas - y esto es independiente de las formas particulares de familia (Rawls, 2001: 163). Obviamente, en este punto, Rawls planeaba discutir formas familiares más allá de la composición del hombre y la mujer, situando familias monoparentales directas (madre o padre) o indirectas (abuelos, tíos, hermanos o parientes cercanos), así como parejas del mismo sexo e incluso en composición monógama o no.

La familia tiene una fuerte influencia social y moral en los niños y jóvenes, y en el contexto de políticas que implican algún elemento afirmativo, este punto es crucial para su éxito y adhesión. Así, la familia señala el desarrollo de las bases para la estructura y el éxito de las políticas que sitúan la no depreciación de las políticas dentro de grupos desfavorables, a fin de que se pueda tener posibilidades de desarrollo social.

En la lectura de Rawls, la familia es un espacio privado al que no se llega con principios políticos que se generalizan para conformarla sino que, por otro lado, se necesitan bases restrictivas que protejan los principios básicos de justicia para los individuos que la componen (Rawls, 2001: 163). Esto significa que Rawls admite que los arreglos familiares tienen autonomía para decidir sobre aspectos de la estructura política en este espacio que no necesariamente son consistentes con las bases de las instituciones derivadas de la toma de decisiones según una posición original, desde que estos aspectos no restrinjan los derechos y libertades de miembros de la familia. El conflicto es claro si destacamos, por ejemplo, una estructura política familiar que im- 
pide que las mujeres trabajen, lo que afecta oportunidades, autonomía y capacidades.

Por otro lado, no hay forma de tener una división clara entre los principios de justicia aplicados a las relaciones sociales en el espacio público que orientan la resolución de problemas de desigualdades y los conflictos en el espacio privado que se disocian de estos principios de justicia. Esto sería entender que estas esferas de la vida no se articulan ni se producen entre sí. La estructura familiar produce y reproduce bases morales, políticas y económicas que impactan las relaciones sociales, es decir refleja una construcción orgánica de la estructura social (por lo tanto, entender a la familia como un «espacio» aparte de la sociedad es inadmisible). Según Rawls (2001: 163), todos los hombres y mujeres adultos miembros de una familia son ciudadanos y esta es su condición básica dentro de la sociedad, por lo que ninguna asociación o institución puede violar sus derechos como tal y, si hay una violación, significa que hay diferencia entre los ciudadanos, con la creación de niveles de ciudadanía.

La debilidad del argumento de Rawls radica en su comprensión sobre la composición de la estructura familiar, precisamente, en el punto en que incorpora a las mujeres. En primer lugar, Rawls asume que existe una desproporción histórica entre hombres y mujeres en la división sexual del trabajo y, sin embargo, se limita a señalar el cuidado únicamente en las actividades del ámbito familiar (Rawls, 2001: 167). Pero no podemos saber si Rawls tenía la intención de considerar otras actividades domésticas o si entendía el cuidado como la única actividad, y no sería apropiado suponerlo en base a una obra inconclusa. El segundo punto es más crítico y también se presenta incompleto: aun en la división sexual del trabajo, Rawls separa el trabajo productivo del trabajo reproductivo dentro de la estructura familiar. Sin embargo, no podemos determinar si separaba actividades productivas de reproductivas en la lógica del valor de uso y de intercambio. Si las separase, podríamos aplicar la lógica de Delphy (2016) sobre la mercantilización de las actividades; si no lo hiciera, podríamos entender que el autor pretendía considerar la división sexual del trabajo más allá de las actividades de cuidado, cubriendo también las actividades domésticas no remuneradas que computan las condiciones materiales de otros trabajos. Pero esto es una incógnita desde la perspectiva rawlsiana.

\section{La ponderación de Nancy Fraser y el trabajo no remunerado}

Fraser, refiriéndose a la «crisis del cuidado», observa las contradicciones en el ámbito de la reproducción social que se intensifican en la sociedad capitalista. Para la autora, una nueva forma de sociedad capitalista que no tiene en cuenta la reproducción social configura una crisis aún mayor. El cuidado, en su perspectiva de crisis, es sistemático en la estructura del orden social, considerando que la acumulación de capital situada por el cuidado se debilita en la dinámica de la reproducción social, en el sentido de que la acumulación demanda no remuneración para la reproducción y, cuando esto 
comienza a exigir una remuneración, los costos de acumulación comienzan a verse afectados; de ahí la crisis (Fraser, 2016: 100).

En la concepción de la crisis, Walby pondera sobre su compresión con la comprensión del género como una relación social y no como un atributo fijo; y así, un régimen de género es un patrón de relaciones de género. En relación con el aspecto económico, por ejemplo, como los patrones implican diferentes grados de familiarización (es decir, estructuras de dependencia y relaciones de poder), la desfamiliarización disocia las dimensiones de dependencia y subordinación en las relaciones. La noción de familiarización involucra procesos en los que las bases familiares se desarrollan a través de elementos de la estructura social que inciden en la organización interna del espacio familiar (en términos políticos, sociales, económicos e institucionales) y externos a las dinámicas sociojurídicas dentro del ámbito del Estado. Estos elementos, entendidos desde el punto de vista de género, implican la comprensión de las desigualdades de género en esta familiarización (Walby, 2015: 27-29). Los análisis pensados podrían situar diferentes formas de entender el fenómeno familiar, específicamente, cuando hablamos de ingresos, trabajo y tiempo, donde vemos el acceso al Estado a través de regulaciones, así como arreglos privados dentro y fuera de las bases familiares.

El sistema capitalista depende de las actividades de reproducción social, en particular, de cómo se sitúa la economía capitalista en una especie de uso implícito de las actividades de cuidado y reproducción de los lazos de afecto; muchas de estas se producen fuera del mercado y, en general, sin remuneración por el trabajo, siendo necesarios para el trabajo remunerado como un trasfondo indispensable para la producción económica en la sociedad capitalista. Según Fraser (2016: 103-106), desde la era industrial existe la separación entre las dos esferas, donde, por un lado, tenemos la producción económica asociada a los hombres y remunerada financieramente $y$, por otro, está la reproducción social que se sitúa en las mujeres y es moralmente remunerada por el amor y la virtud; creando una esfera doméstica que invisibiliza los procesos de desigualdad, y guiando las relaciones crónicas de poder y jerarquía. Por lo tanto, hay una relación de dependencia marcada por la desestructuración mutua y constante de las esferas.

La producción y la reproducción también se ven afectadas por la crisis y no deben simplificarse. Así, Walby (2015: 73-77) indica que, en el contexto de las mujeres no hay ningún proceso de incorporación de familiarización, dejando la producción y volviendo a la reproducción. Es decir, que las mujeres, ya sea en caso de pérdida de trabajo u opción por no tener un trabajo remunerado, que se indique efectivamente su retorno a la exclusividad de la reproducción. Por lo tanto, la autora separa los regímenes de género en domésticos y públicos (Walby, 2015: 148), siendo que este último también presenta dos formas: neoliberal y socialdemócrata, y sus instituciones también difieren.

Las principales diferencias recaen en la generificación de la democracia y el grado 
de desigualdad de género, donde tenemos diferentes vías de transición entre regímenes domésticos anteriores a regímenes públicos de género. En ellas el trabajo, los ingresos, la autonomía, el cuidado y el trabajo no remunerado, los servicios prestados por el Estado y sus relaciones democráticas como acceso y disputa son procesos de participación democrática, en particular en lo que respecta a la relación de estos aspectos mediados por el Estado (socialdemócrata) o por el mercado (neoliberal).

Delphy señala que hay literatura integral que sitúa las tareas domésticas como trabajo, pero no pagado, con lo cual se interrumpe la lógica de que el trabajo implica reconocimiento y valorización directa. Delphy (2016: 8o-84) sigue el razonamiento económico del trabajo doméstico como una actividad especializada, que consume ciertos bienes para producir derivaciones para una lógica pro mercado. Es decir, la familia produce bienes que no se restringen al consumo familiar pero que son capaces de lograr el consumo externo; por ejemplo, cuidar los alimentos producidos en la familia permite restituir la energía que se consumirá fuera del entorno familiar (y esta energía valorada en el entorno familiar no se incorpora a los costes de producción en el sistema capitalista). En esta lógica, a menudo se afirma que el trabajo doméstico no es remunerado por los costos y la no valoración en la base del mercado.

En el sistema de libre competencia capitalista del siglo XIX, los imperativos de producción y reproducción son directamente contradictorios. Observamos el trabajo extremadamente barato de las mujeres en las fábricas, con crisis en la reproducción social y una «destrucción de la familia» en el contexto del desorden social, que resulta en mecanismos de "protección» de las mujeres, idealizando la casa, la familia y el hogar; cuyo conflicto arraiga la dominación masculina, el imaginario de género centrado en esferas separadas y la mujer como «ángel de la casa» (Fraser, 2016: 105-108). Al mismo tiempo, el ideal de domesticidad incide sobre las diferentes condiciones de las mujeres, en la minoría legal y la dependencia institucionalizada.

En el sistema de capitalismo de Estado del siglo XX, la contradicción permanece, sin embargo, con el Estado junto respecto a la reproducción, a través de la «responsabilidad pública por el bienestar» que contiene los efectos corrosivos de la explotación y el desempleo masivo (Fraser, 2016: 108-112). Vemos al Estado crear las condiciones para salvar al sistema económico de su autodesestabilización a través de inversiones públicas con la internalización de la reproducción social dentro del dominio del orden capitalista. El trabajo y el consumo están unidos en la figura del trabajador; este, todavía masculino y de carácter público, donde la casa, aspecto privado, es el centro del consumo privado, síntesis de la mercantilización y protección social. Dichos elementos entraron en las concepciones de las demandas por democracia y justicia social, estabilizando la reproducción social. Sin embargo, un sistema periféricamente excluyente, racializado y clasista en el trabajo reproductivo, productivo y de acceso al mercado y al Estado, con un claro sacrificio de emancipación por una alianza de protección social y mercantilización, incluso con sus efectos negativos mitigados. 
La vía socialdemócrata sitúa la movilización de las fuerzas políticas en contexto de la comunidad democrática, en donde estos contextos públicos y políticos son creados con el objetivo de construir condiciones equitativas para las mujeres; la vía neoliberal apunta a la mediación por parte del mercado, la producción, el consumo y las mercancías, acoplando servicios, en un primer momento públicos, como mercancías, además de la lógica de socialización por el empleo y mercado laboral (Walby, 2015: 153-154).

Así, para Walby, un modelo debe incluir más allá de la familia y el Estado de bienestar social, y debe tener una base: económica, política, violencia y sociedad civil. En estos dominios institucionales tenemos una serie de prácticas en las que se reproduce la vida social, por lo tanto, los regímenes de género tienen un carácter relacional, especialmente con el capitalismo, como mutuamente adaptativo en un sistema complejo que da forma a ambos (Walby, 2015: 153). Así, el cambio no es un proceso simple o lineal, sino impregnado de una reestructuración que afecta a la vida social.

En el sistema de capitalismo globalizado financieramente, vemos la incorporación de las mujeres al mercado laboral y la agenda neoliberal de la desinversión en el bienestar social, impactando en la realización del cuidado en las familias, donde colocamos la externalización del cuidado en términos de la capacidad para comprarlo o no. Y sin el salario familiar al que se refiere Fraser (2016: 111-112), tenemos el modelo de «dos proveedores familiares». El sistema de deuda de los Estados configura la necesidad de acuerdos y reducciones fiscales, imposibilitando la inversión en el orden de la reproducción y protección social, sustituyendo a la oferta por los agentes privados en el mercado.

En la cuestión del crecimiento económico, el modelo neoliberal es desafiado en la relación entre el empleo integral y la dimensión del cuidado, generalmente a cargo de una mujer. Está relacionado con el crecimiento, la distribución justa, ambos son vistos como compensación (Walby, 2015: 155-156). La combinación del crecimiento con una distribución justa es esencial y necesita que se rompa su dimensión de género. Al interrumpir, la inestabilidad afectada por la economía se ve afectada, incluso democráticamente en las oportunidades, invirtiendo lógicas de trabajo, cuyo carácter de figura es masculino. Además, rompiendo la lógica, hemos situado el agotamiento de los modelos de austeridad que comercializan y privatizan elementos del orden público que estabilizarían los proyectos de vida y los contextos sociales.

La demanda por el reconocimiento y la emancipación se incorporó por el mercado en la forma de oferta de puesto de trabajo (aún racializada, generificado y clasista) y ya no de garantía de derechos y seguridad social (Fraser, 2016: 113-114). La lógica de la equidad abarca únicamente la producción y no la reproducción, que aparece con residuos, asumiendo una nueva intensidad. La mercantilización del cuidado y de la reproducción social termina «ofreciendo» medios (trabajadoras del hogar, cuidadores, servicios especializados, entre otros) a los que impone un choque entre los 
que pueden y los que no pueden permitirse el pago, como en el siglo XX y en la era industrial, la diferencia es que ahora no hay manera de garantizar la presencia del Estado en condiciones inviables de "comprar».

Para Fraser (2016: 113), lo que surge de esta crisis es la reconfiguración de actores y procesos, lo cual es recurrente en el sistema capitalista cuando la situación agoniza. Y esta crisis no se resolverá con la política social, a no ser que ocurran cambios estructurales y profundos del orden social, eliminando la subyugación de la reproducción a la producción, sin sacrificar la protección social ni la emancipación, lo que requiere reinventar la distinción producción-reproducción y reimaginar el orden de género.

Algunos encuentros pueden ocurrir cuando tales cambios alcanzan a la familia nuclear. Las nuevas formas de producción y reproducción social se presentan en un entorno más inestable para el empleo y reducidas a las familias, estableciendo un mayor grado de inestabilidad e incertidumbre, ¿qué tipo de Estado de bienestar social se configura aquí? Fraser (2013: 120-123) señalará la inevitabilidad de la reconfiguración del cuidado basado en una propuesta: el cuidador universal. La construcción de la autora se basa en el entendimiento de que la única manera de tener justicia de género es considerar los patrones de vida de las mujeres como patrón normativo. No obstante, observamos que los modelos anteriores se basan en la lógica masculina del trabajo y de la consideración sobre el trabajo doméstico (Fraser, 2013: 133-135).

La propuesta de la cuidadora universal desmantelaría la oposición que no observa las relaciones desiguales de género entre el cuidado y la provisión, integrándolas y superando el orden de género jerarquizante y silenciador de las mujeres, en particular, los trade-off que forman parte de los procesos de producción y reproducción social. Nancy Fraser señala siete principios que involucran a este cuidador universal, a saber: antipobreza, antiexplotación, igualdad de ingresos, igualdad en el tiempo libre, igualdad de respeto, antimarginación y antiandrocentrismo. Cada trade-off implica una elección, una decisión y un costo vinculado y, agregándolos, existe la posibilidad de reducirlos (Fraser, 2013: 133-137). Finalmente, la justicia requeriría la deconstrucción de género (la cual, según Moller, se manifiesta en el mismo sentido, sin embargo, insistiendo en el fin del género).

\section{El trabajo doméstico: Entre la invisibilización y la negación}

El significado del trabajo doméstico y el cuidado implica una serie de configuraciones que se movilizan en torno a construcciones políticas y demandas. En el contexto de la atención podríamos considerar el distanciamiento del amor materno naturalizado y la incorporación de una medida de derecho social y justicia que evoque la dimensión de la ciudadanía.

En este contexto, Bila Sorj aduce que las feministas liberales y las feministas anticapitalistas no ven la relación del reconocimiento de las cuidadoras como trabaja- 
doras. Esta incapacidad no les permite reconocer algunos elementos, comenzando por el trabajo doméstico remunerado, responsable de una inserción significativa de la mujer en el mercado laboral, tanto en el trabajo directo como en la contratación de otras mujeres (Sorj, 2014: 123-124). En este caso, la politización tardía de los derechos de las trabajadoras domésticas sitúa la jerarquía de género y clase en los intereses laborales, lo que afecta a este trabajo ya no como puerta de entrada, a pesar de que las configuraciones de trabajo en la legislación hayan sido constantemente burladas en lo que dice la ley.

Delphy utiliza los términos ocupación y clase para referirse a las mujeres dentro de los estudios de estratificación, en los que se presentan las estrategias para apuntar a las mujeres dentro de los criterios de producción y trabajo. Para la autora, las mujeres no forman parte del mismo sistema de diferencia que los hombres (Delphy, 2016: 8587). Los criterios llevan a las mujeres a ser clasificadas por la ocupación de los maridos, lo que impide la comparación entre hombres y mujeres, además de degradar a la mujer. Esta elección metodológica y teórica trae distorsión en el análisis comparativo, además, invisibiliza su posición social.

En los tejidos de las relaciones de poder vemos a los grupos dominantes organizar patrones de necesidades que son interiorizados por los dominados. Estos patrones no replican la superación de la condición de subordinación, además de que la necesidad inscrita aquí es puntual e incapaz de revertir lógicas o llegar a los sujetos, es ahí donde reposa la separación entre las esferas política, económica, doméstica y personal (Fraser, 2013: 65). Las necesidades impregnadas en las dimensiones materiales de las relaciones de producción no son las mismas que las situadas en la dimensión de reproducción, así como que sus lógicas de apropiación son diferentes en cada ámbito; la cuestión planteada es la desconexión deliberada e incluso la reificación, de modo que estos contextos interrelacionados entre las esferas no se perciben como tales. La percepción de estos como interrelacionados apunta a la superación de los mecanismos de subordinación, ya que considerando que la estructura de dominación es sistémica, los esfuerzos de ruptura podrían desarrollarse mejor. En este punto, Fraser (2013) señala que las justificaciones de este modelo separado consisten en, al menos, considerar los procedimientos y la competencia, así como sus consecuencias, alternando con instrumentos distributivos, e instalando una «democracia» y supuesta «igualdad».

Delphy señala un grave fracaso en este proceso, que oscurece la presencia o ausencia de la dependencia económica de mujeres hacia hombres, donde una mujer se halla en una posición inferior a su marido (Delphy, 2016). Las mujeres casadas y desocupadas son socialmente alienadas por la «asociación con el matrimonio» como criterio. ¿Existe una idea de clase aquí que apunta a que alguien desocupado no debería ser clasificado por sí mismo, o sea sin existencia social? ¿Una clara invisibilidad? No tener una ocupación es no tener una posición social, así como no hay una situación económica. 
El lugar estratégico del trabajo doméstico remunerado apunta a la invisibilización de la participación masculina respecto a su división sexual, donde la sobrecarga de la mujer se intensifica y las experiencias sociales diversificadas impactan en el mismo sentido. Bila Sorj (2014: 126) destaca una posición ambivalente en el trato con cuidadoras: en la defensa de la igualdad de trato doméstico hay desconfianza, en la medida en que se sublima la posibilidad de abolición del trabajo doméstico y la desvalorización de sus competencias, creando tensiones en la lucha por una justicia distributiva.

Delphy asume el consenso como una de las principales funciones de la familia moderna, de hecho, como su última función distante dentro del orden económico (Delphy, 2016: 42-43). En este contexto se cuentan las instituciones capturadas (como matrimonio, familia, maternidad, cuidado, responsabilidad familiar), donde las familias juegan un papel en la sociedad. En el caso del consenso, podemos ver las relaciones de producción y consenso, las exclusiones e inclusiones en el orden social. El consenso sería constitutivo de la estructura familiar, que repercute en el estatus, percibido tanto interna como externamente en relación con la familia. Hay un error al considerar a la familia como un sistema homogéneo y como un único objeto de consenso.

Por lo tanto, la idea de Delphy es considerar a la familia como un espacio distributivo, tanto en términos cuantitativos como cualitativos, donde se percibe la influencia masculina (del proveedor) en la recepción de las fracciones más grandes y mejores de oportunidades del desarrollo del plan de vida, trabajo, ingresos, acceso a la educación y servicios públicos (Delphy, 2016: 48-49). Incluso la «elección» de la comida tendría un componente socialmente generificado, al igual que el alcohol. El acceso y el alcance tienen una base diferenciada y regulada en la prohibición, así como el componente moral involucrado en el consumo de alcohol. Delphy señala algunos estudios naturalistas que sitúan la teoría de las necesidades físicas, que pondrían más fuerza, peso e importancia en el trabajo masculino como si fuera más agotador que el trabajo doméstico. Vemos de nuevo la lógica del trabajo y de las acciones del cuidado pensadas en la lógica masculina, como señalan Moller (1989: 145) y Fraser (2013: 215-216).

\section{El cuidado y las actividades domésticas en las políticas públicas}

El cuidado en Brasil aún no tiene una perspectiva normativa clara en sus políticas públicas o en sus dispositivos constitucionales o administrativos regulados. Sin embargo, se entiende que las políticas relacionadas con el cuidado básico, el cuidado de los niños y los ancianos, están vinculadas a los servicios de salud pública dentro del Sistema Unificado de Salud (SUS). El artículo 6 de la Constitución brasileña destaca que los derechos sociales son «educación, salud, alimentación, trabajo, vivienda, transporte, ocio, seguridad, seguridad social, la protección a la maternidad y a los niños, así como, la asistencia a los indefensos», por lo que la asistencia, protección, 
seguridad y seguridad social son ópticas que amparan la perspectiva que puede ser asociada a la dimensión del cuidado.

En el caso de las políticas públicas para las mujeres, el proceso legislativo brasileño involucra disputas que van más allá de las concepciones técnicas y políticas de cómo producir una política y generar sustento para su institucionalización, abarcando así confrontaciones normativas y concepciones de género sobre el concepto de mujer y sus condicionantes sociales (Ferreira, 2021b: 201-204). Así, en una dinámica de enfrentamientos, se evoca el proceso de reconocimiento de derechos como medida política de organización de lineamientos sobre un tema social reivindicado, en este punto, el cuidado, que es igualmente confrontado en su base normativa y conceptual capaz de agregar esquemas claros para la aplicación integral de los derechos (Ferreira, 2021c: 137-140). Sin embargo, las disputas legislativas están guiadas por la dinámica entre autonomía y agencia imperfecta, es decir, una gramática moral que producirá una legislación que partirá en concepciones integrales o limitadas, debido a la fragilidad legislativa, poco adherente a las reivindicaciones de los derechos de las mujeres (Ferreira, 2021a: 415).

Guimarães, Hirata y Sugita (2011: 156-160) señalan que el concepto de cuidado aún está constituido en un sentido derivado que debe ser relacionado a la asistencia y seguridad social, colocando un conjunto de medidas públicas imprescindibles para el bienestar de la población en un Estado de bienestar; además, desde la perspectiva francesa, se considera la sensación de inquietud afectiva para el otro. Asimismo, la visión japonesa considera un cuidado a largo plazo, asociada al cuidado de los ancianos o personas en estado de dependencia. Y, por su parte, en el caso brasileño se asocia las nociones de «cuidar» o de «encargarse» de las personas, al hogar, y a ciertas situaciones que apuntan a una atención específica. Para las autoras, el cuidado está asociado con la cuestión del género, mientras que esta actividad se naturaliza como inherentemente femenina. Para Araujo (2019: 7) el cuidado es una técnica y no una característica intrínseca y el distanciamiento emocional y personal es parte del procedimiento técnico, creando barreras al sacrificio y altruismo, distanciando las relaciones de dadivas e intercambio.

Nuestro objetivo, en este trabajo, no es debatir formas de atención privada, sino señalarlas desde la perspectiva de la administración pública como una actividad del Estado en términos de construir bases más justas en el ámbito de las actividades domésticas y que incidan directamente en la posibilidad de equidad de las condiciones de justicia en relación con la mujer. Por lo tanto, nos referimos únicamente a los instrumentos normativos del Estado brasileño, observando al menos cuatro de estos.

En consulta con el sitio web de la Cámara de Diputados y el Senado Federal de Brasil, encontramos alrededor de 716 instrumentos - proyectos de ley, propuestas de enmiendas a la Constitución y requerimientos- que implican el término «cuidado", y que abarcan desde mejoras en las condiciones de los trabajadores en el área de la 
salud, hasta políticas específicas para ancianos, niños y personas en situaciones de dependencia físico-motora, sicosocial o mental.

Específicamente, encontramos dos proyectos de ley que observan una Política Nacional de Cuidado. En el artículo segundo del proyecto de ley 2029/2015, ${ }^{1}$ ya archivado, destinado principalmente al cuidado a largo plazo, el cuidado fue descrito como

un conjunto de acciones integradas, encaminadas a cuidar y promover el bienestar, la salud, la seguridad, la autonomía y la independencia de las personas, en consideradas condiciones, las limitaciones y las necesidades personales, familiares, culturales, económicas, sociales y comunitarias; respetando la individualidad y la dignidad humana.

Esta política se habría aplicado de forma descentralizada y articularía a la Unión, los estados, el Distrito Federal y los municipios, teniendo los siguientes principios, expuestos en su artículo 50:

- Respeto a la dignidad inherente, a la autonomía y a la independencia de las personas, inclusive para tomar sus propias decisiones.

- Derecho a la convivencia familiar y comunitaria.

- Valorización y respeto por la vida, la ciudadanía, las habilidades y talentos de las personas.

- Cuidado humanizado e individualizado, en respeto a las características sociales, culturales, económicas, los valores y preferencias de las personas.

- Respeto a las diferencias de origen, raza, edad, nacionalidad, género, orientación sexual y religiosa.

Entre sus directrices, en el artículo sexto y, específicamente, en el inciso segundo, vemos la responsabilidad de los poderes públicos para la elaboración y financiación de un sistema articulado y multidisciplinar de atención y apoyo a las personas que necesitan cuidado profesional, familiar o comunitario, y uno de sus objetivos en el artículo 7 , inciso segundo, es asegurar el desarrollo de una red articulada, integrada e intersectorial de cuidado; así como en el inciso decimosexto, el de garantizar la protección, la inclusión profesional, la seguridad, la salud y el bienestar del cuidador formal, informal y comunitario, especialmente el cuidador de edad avanzada o en situación de vulnerabilidad social, y en el punto decimoséptimo, el de difundir la cultura del cuidado. Señalamos que el artículo 8 prevé la ley presupuestaria para la constitución y mantenimiento de programas y proyectos dirigidos a la Política Nacional del Cuidado.

1. Proyecto de ley 2029/2015, disponible en https://bit.ly/3orcVny. 
En el caso del proyecto de ley 5791/2019, ${ }^{2}$ que está en la mesa directora de la Cámara de Diputados y a la espera de tramitación para las comisiones temáticas, también se ocupa de la Política Nacional de Cuidado. En este se interpreta el cuidado de la siguiente manera, a través del artículo 2 :

A los efectos de esta Ley, el cuidado es el conjunto de acciones interdisciplinares encaminadas a promover el bienestar, la salud, la seguridad, la autonomía y la independencia general de la persona en situación de dependencia para las actividades de la vida cotidiana, teniendo en cuenta sus necesidades personales, familiares, educativas, profesionales, sociales, culturales y comunitarias, su individualidad y dignidad inherente.

Los principios del proyecto de ley 5791/2019 son similares al proyecto de ley 2029/2015; sin embargo, en el inciso sexto del artículo quinto, añade como principios el «respeto y valorización del cuidador profesional, familiar, social y comunitario». Destacamos que, entre las directrices previstas en el artículo 6 tenemos, en su inciso sexto, el «ejercicio de control social de la oferta de bienes y servicios y de informaciones necesarias para el cuidado" y en su inciso séptimo, la "prestación de servicios en equipos sociales cercanos o en el domicilio de la persona necesitada de cuidado, incluso en la zona rural». Un punto culminante para el objetivo de la política es el artículo 7 , inciso decimosegundo, que se refiere a la promoción de espacios intersectoriales locales para la articulación de acciones y la elaboración de planes de acción conjuntos centrados en las personas que necesitan de cuidados y sus familias, con la participación de profesionales de la salud, de asistencia social, de educación y de órganos para la promoción, protección y defensa de los derechos. Asimismo, el artículo 8 , que señala la intersectorialidad de las políticas públicas en las áreas de la asistencia social, la salud, la educación, la vivienda, el trabajo y la renta, la cultura, la seguridad social, el deporte, el ocio, la protección y la garantía del derecho.

Por último, tenemos la Propuesta de Enmienda a la Constitución 348/2017, ${ }^{3}$ que incluye la atención a largo plazo de los derechos que conforman la seguridad social y se encuentra para consideración del plenario del Congreso. Esta enmienda incluiría los cuidados a largo plazo en la propuesta de presupuesto de la seguridad social, y los definiría como aquellos «prestados a las personas con pérdida de autonomía funcional que se encuentran en una situación de dependencia para el ejercicio de las actividades de la vida diaria», siendo permitida la iniciativa privada, observando el cumplimiento de las normas relativas a los cuidados a largo plazo emitidas por las autoridades públicas.

2. Proyecto de ley 5791/2019, disponible en https://bit.ly/3qIJbop.

3. Propuesta de Enmienda Constitucional 348/2017, disponible en https://bit.ly/3cpoACV. 
No debemos olvidar la Enmienda Constitucional 72 de 2013, ${ }^{4}$ que trajo derechos laborales para los trabajadores domésticos y otros trabajadores urbanos y rurales, como la indemnización compensatoria en casos de despido, seguro de desempleo, fondo de garantía de tiempo de servicio, salario mínimo, base salarial, irreductibilidad y garantía salarial, decimotercer salario, remuneración del trabajo nocturno superior al diurno, salario familiar pago por dependiente del trabajador de bajos ingresos, descanso semanal remunerado, disfrute de vacaciones anuales remuneradas, protección del mercado laboral de la mujer, entre otros derechos. Aquí, aparece claramente la perspectiva de valorización de las actividades domésticas, vinculadas a la ocupación de una empleada doméstica.

Lo que vemos en el ámbito de las propuestas de políticas públicas en Brasil es una organización de acciones que consideran la perspectiva del cuidado como una medida de Estado a largo plazo, que apunta a mejoras en los campos de la dignidad y la igualdad como partes de un programa de desarrollo social, considerando el contexto. Especialmente el proyecto de ley 5791/2019, que se ocupa de las capacidades de las personas, es decir que la política de cuidado es una acción del Estado que permite no solo potenciar el desarrollo de los proyectos de vida individuales, sino que, además, estos apunten en una dimensión social de pertenencia a la ciudadanía y desarrollo del país, lo que se observa en la Constitución brasileña de $1988.5^{5}$ Se trata de objetivos ambiciosos, sobre todo teniendo en cuenta la negligencia histórica de los servicios de salud en el contexto brasileño, especialmente en relación con los ancianos y las personas en un estado de dependencia momentánea o permanente.

Otro aspecto para tener en cuenta es que las llamadas políticas de cuidado aún no observan la dimensión del trabajo de cuidado familiar, que no se paga y que necesita de atención. Situar los equipos públicos de cuidado es crítico en esta perspectiva. Planificar redes y acciones integradas entre las esferas institucionales brasileñas no es lo mismo que señalar claramente qué equipos de cuidado son primordiales para el desarrollo de una política nacional. De hecho, como una ley nacional que debe salvaguardar las condiciones financieras y político-institucionales infraconstitucionales, la misma no podría interferir en la autonomía relativa de los estados y municipios; sin embargo, poner ciertos centros de equipos públicos de cuidado no es un impedimento para la legislatura constitucional.

Al mismo tiempo, vemos la fuerte incidencia de la neutralidad de género en la observancia de las políticas de cuidado: no vemos en las justificaciones de los proyectos las indicaciones de que el sexo femenino es más sobrecargado en las actividades de cuidado y las tareas domésticas, así como en relación con la baja remuneración de estas actividades. El sujeto de justicia previsto en estos proyectos de ley y enmienda

4. Enmienda Constitucional 72, 2 de abril de 2013, disponible en https://bit.ly/2ZTojsa.

5. Constitución de la República Federal de Brasil de 1988, disponible en https://bit.ly/3Cqv4PA. 
constitucional son aquellos que recibieran la actividad de cuidado y no necesariamente los que la practican; a pesar de que estos últimos son observados como sujetos de justicia, están mal enmarcados desde la perspectiva de Fraser (2009: 12-20; 2013: 189-197), siendo más desvalorizados por su trabajo ser configurado como sin género, así como tales proyectos presentan la noción de trabajo esencialmente profesional, sin tener en cuenta los contornos que incluyen a la familia en este trabajo, desde la perspectiva de Delphy (2016: 67-69).

\section{Consideraciones finales}

Este trabajo buscaba desarrollar una crítica teórica sobre la familia, el cuidado y el trabajo doméstico, su no remuneración y las condiciones de justicia vinculadas a sus perspectivas. Tomamos como base las críticas de Susan Moller y Christine Delphy, cuyas nociones sitúan la generificación de las relaciones productivas y reproductivas dentro de la familia y cómo esto se extiende a toda la sociedad. Sin embargo, no podíamos hablar de producción y reproducción solo como elementos dados, en particular, a la esfera pública y privada, respectivamente. Por el contrario, como se ha dicho, la familia, la sociedad, el Estado y el mercado son instituciones y procesos que impregnan los dos sistemas y forman un conjunto que, en definitiva, explota y expropia la fuerza de trabajo de las mujeres, a diferencia de los hombres. Cuando se observan las dimensiones de las actividades domésticas y las actividades de cuidado se evidencia una clara relación de explotación.

Las perspectivas de igualdad de justicia en John Rawls y Ronald Dworkin enfatizan los procedimientos y procesos, y dan poca atención a la familia como institución. Dworkin la considera mínimamente y Rawls presta atención a la familia, a pesar de que no desarrolló completamente su crítica. Este último, sin embargo, busca disipar la noción de que la familia no sería un espacio para la justicia, porque no se centra en las relaciones de consumo y la disputa de recursos escasos; una lógica iniciada con Aristóteles y refinada por David Hume. La familia, como espacio de amor, los lazos de efectividad y del cuidado, no está impregnada de relaciones de poder, cobranza y disputa de bienes, considerando que lo que se hace es en nombre de algo mayor: el mantenimiento de la familia, por lo tanto, la justicia es algo que no se concibe. Rawls está fundamentalmente en contra de este pensamiento y afirma: la familia, como institución, no debe ser inmune a los procesos que violan los derechos básicos de libertad, igualdad y oportunidades justas.

Considerar el trabajo doméstico y el cuidado desde la perspectiva de la justicia significa considerar las condiciones sociales, políticas, institucionales y económicas que impregnan sus configuraciones históricamente dadas. El trabajo no remunerado expropia fuerza de trabajo e impide la igualdad de trato y desarrollo de planes de vida, así como el acceso a espacios sociales que requieren tiempo, recursos y contac- 
tos. Al mismo tiempo, no se debe caer en la retórica de que la remuneración es suficiente para resolver esta cuestión, dado que la oferta de trabajo doméstico y cuidado por parte del mercado no significa necesariamente una valoración coherente, ya que puede seguir la lógica masculina del trabajo.

En consecuencia, las políticas públicas creadas $-\mathrm{y}$ aquí presentamos dos proyectos de ley, una propuesta de enmienda a la Constitución y una enmienda ya sancionada - sitúan las configuraciones y lecturas del trabajo doméstico y el cuidado desde una perspectiva neutra de género, desvinculada de la contextualización que indica que las mujeres son las principales protagonistas de estas actividades, con bajos salarios y un alto grado de inestabilidad.

Aquí vemos la lógica de las propuestas que se centran en una dimensión que organiza solo una parte de aquellos sujetos de justicia de una relación que es claramente desigual, esto es, que no incorporan las dimensiones de las desigualdades. La lógica que desprecia las desigualdades acaba ignorando que, en el ámbito familiar, algunas personas (mujeres en particular) acaban asumiendo la responsabilidad de la producción y reproducción sin que su esfuerzo o trabajo se incorpore debidamente al sistema social, económico y político en la consideración de los principios de justicia. ¿Es esta la dimensión del sacrificio, generalmente asociada con el cuidado y a las actividades domésticas? ¿Es esto una institucionalización del sacrificio donde los ofertantes del cuidado son elevados a la dimensión del reconocimiento del Estado y, sin embargo, son aún inferiores como pares en la justicia social?

La perspectiva presentada por los proyectos de ley se distancia de la consideración de pares de interacción social, es decir, personas que deben ser consideradas como sujetos de igual valor moral en la lógica de la interacción (Fraser, 2009: 16-18). Aunque se coloca la óptica de posibilidad de igual ejercicio de capacidades en el contexto familiar y de la existencia de oportunidades para los miembros dependientes de la familia, Nussbaum (2006: 98-103) considera que la dimensión de las capacidades (ejercitar habilidades para desarrollar oportunidades) debe construir perspectivas igualitarias de los sujetos, pero sin reducir necesariamente la de otras, Rawls (1971: 620-630; 1993: 466-467; 2001: 168-170) y Dworkin (1985: 41-50) se adhieren a esta colocación. Esta sería una interpretación directa y aplicada de la base rawlsiana de que la no consecución de los principios de justicia no debe reducir el ejercicio de los derechos y libertades de los miembros de esta asociación familiar. No observar la desigualdad de género como constructor de desigualdades sociales a un nivel más amplio (Moller, 1989: 16-17), apunta a desigualdades aún más incidentes, teniendo en cuenta que los sujetos afectados incorporan una alta carga.

Por lo tanto, los proyectos de ley deben considerar el cuidado en su dimensión de género, raza y clase, señalando mecanismos claros que potencialicen la perspectiva social de la justicia. En este punto, los equipos de cuidado bien definidos, la regulación de la profesión y sus derechos, la red articulada con servicios públicos y las in- 
versiones paralelas al mercado y su base privada, así como una delimitación del contexto social territorial brasileño, deberían ser parte de los debates de estos proyectos, teniendo en cuenta que no solo son políticas públicas de "cuidado», al contrario, son políticas de desarrollo social, que apuntan sujetos de justicia y derechos humanos, al tiempo que se centran en el potencial de los individuos. Lo que vemos, por último, en estos proyectos de ley, es insuficiente para la construcción de los sujetos de justicia y sus contextos conexos.

\section{Referencias}

Araujo, Anna Bárbara (2019). "Gênero, reciprocidade e o mercado de cuidadores de idosos». Revista Estudos Feministas, 27 (1): 1-13. Disponible en https://bit. ly/3FjpkZW.

De Vita, Álvaro (1993). Justiça liberal: Argumentos liberais contra o neoliberalismo. Río de Janeiro: Paz e Terra.

DwORKIN, Ronald (1985). A matter of principle. Cambridge: Harvard University Press.

Ferreira, Vinicius (2021a). «Planejamento familiar: inimigo a ser combatido, aliado libertador ou falso amigo?». Revista Brasileira de Políticas Públicas, 11 (1): 394-417. Disponible en https://bit.ly/3HlGkAu.

-. (2021b). «Covid-19, Janelas de oportunidade e políticas para mulheres: análise sobre projetos de lei apresentados no Congresso Nacional». E-Legis, 14: 194-234. Disponible en https://bit.ly/3Fq27Wa.

-. (2021c). «Reconhecimento de direitos de pessoas trans: Alternativas, políticas e ativismo teórico-judicial». Revista de Direito Brasileira, 28 (11): 131-163. Disponible en https://bit.ly/3FkDwBU.

Guimaraes, Nadya Araujo, Helena Sumiko Hirata y Kurumi Sugita. (2011). «Cuidado e cuidadoras: o trabalho de care no Brasil, França e Japão». Sociologia \& Antropologia, 1 (1): 151-180. DOI: 10.1590/2238-38752011v117.

Hume, David (1967). A treatise on human nature. Oxford: Clarendon.

Fraser, Nancy (2009). Scales of justice: Reimagining political space in a globalizing world. Nueva York: Columbia University Press.

-. (2013). Fortunes of feminism. Londres: Verso.

-. (2016). «Contradiction of capital and care». New Left Review, 100: 99-117. Disponible en https://bit.ly/3kJajZA.

Nussbaum, Martha (2006). Frontiers of justice. Cambridge: Harvard University Press. Moller, Susan (1989). Justice, gender, and the family. Nueva York: Basic Books. RAWLs, John (1971). A theory of justice. Cambridge: Harvard University Press.

-. (1993). Political liberalism. Nueva York: Columbia University Press.

-. (2001). Justice as fairness: A restatement. Massachusetts: Harvard University Press. 
SoRJ, Bila (2014). «Socialização do cuidado e desigualdades sociais». Tempo social, (26) 1: 123-128. Disponible en https://bit.ly/3CnzhDF.

Walby, Sylvia (2015). Crisis. Cambridge: Polity.

\section{Sobre el autor}

Vinicius Ferreira Baptista es doctor en Políticas Públicas por la Universidad Estatal de Río de Janeiro. Profesor adjunto del Departamento de Administración Pública de la Universidad Federal Rural de Río de Janeiro. Profesor de los programas de posgrado en Desarrollo Territorial y Políticas Públicas de la Universidad Federal Rural de Río de Janeiro y en Ciencias Políticas de la Universidad Federal del Estado de Río de Janeiro. Su correo electrónico es viniciusferbap@ufrrj.br. (D) https://orcid. org/0000-0002-8717-8332. 
La Revista Chilena de Derecho del Trabajo y de la Seguridad Social es una publicación semestral del Departamento de Derecho del Trabajo y de la Seguridad Social de la Facultad de Derecho de la Universidad de Chile, y que tiene por objetivo el análisis dogmático y científico de las instituciones jurídico-laborales y de seguridad social tanto nacionales como del derecho comparado y sus principales efectos en las sociedades en las que rigen.

\author{
DIRECTOR \\ Luis Lizama Portal \\ EDITOR \\ Claudio Palavecino Cáceres \\ SECRETARIO DE REDACCIÓN \\ Eduardo Yañez Monje \\ SITIO WEB \\ revistatrabajo.uchile.cl \\ CORREO ELECTRÓNICO \\ pyanez@derecho.uchile.cl \\ LICENCIA DE ESTE ARTÍCULO \\ Creative Commons Atribución Compartir Igual 4.o Internacional
}

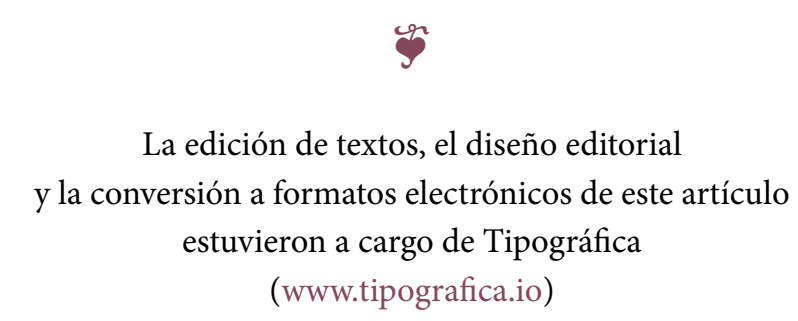

